Research Article

\title{
Ocean Surface Current Observation with a Dual Monopole-Cross-Loop Antenna Array
}

\author{
Yeping Lai, Hao Zhou, Yuming Zeng, and Biyang Wen \\ School of Electronic Information, Wuhan University, Wuhan 430072, China \\ Correspondence should be addressed to Hao Zhou; zhou.h@whu.edu.cn
}

Received 21 July 2017; Revised 7 November 2017; Accepted 22 November 2017; Published 18 December 2017

Academic Editor: Amerigo Capria

Copyright (C) 2017 Yeping Lai et al. This is an open access article distributed under the Creative Commons Attribution License, which permits unrestricted use, distribution, and reproduction in any medium, provided the original work is properly cited.

\begin{abstract}
The high-frequency radars (HFRs) receiving the sea echoes backscattered from the fluctuating ocean surface to remotely sense ocean surface currents are a popular and powerful tool in oceanic observation. Dominant error source in current measurement for HFR systems has been recognized to be the direction of arrival (DOA) determination of the sea echoes. To eliminate this error and therefore improve the performance of direction-finding HFR system in current measurement, we have investigated a dual monopole-cross-loop (MCL) antenna array in current observation. Simulations indicated that the dual MCL antenna array has a better performance than the conventional single MCL antenna system in current mapping, especially for the complex current profile. And comparisons of radar field data and buoy measurements suggested that the RMSE value was larger than $15 \mathrm{~cm} / \mathrm{s}$ for the conventional MCL antenna. But it decreased to $12.64 \mathrm{~cm} / \mathrm{s}$ for the dual MCL antenna array. Moreover, the temporal coverage rate also showed the benefit of using this antenna system in current mapping. The results demonstrated that it is advisable to adopt the dual MCL antenna array in operational applications.
\end{abstract}

\section{Introduction}

The HFRs operating at a frequency range of $3 \mathrm{MHz}$ to $30 \mathrm{MHz}$ have been extensively used to provide ocean surface current in real time [1]. These radars may sense current velocity up to a range of $300 \mathrm{~km}$ from the shore relying on the parameters of the radar configuration. And the data products derived from these radars can be used in many fields, including oceanographic and meteorological researches, monitoring the dispersal of pollution and other floating objects, as well as coastal and harbor management.

The current observation HFRs can be roughly divided into two types based on the method employed to determine the bearing of the radial currents: beam forming (e.g., WERA [2]) and direction finding (e.g., CODAR [3] and OSMAR-S $[4,5])$. Beam forming radars electronically steer a linear phased array of receiving antennas toward a patch of the ocean surface. This type of radar can provide an excellent angular resolution to separate the sea echoes scattered from different patch efficiently but with a cost of occupying a large space in practice, while the direction-finding method is usually adopted by transportable radar systems, which are equipped with a MCL antenna comprising one monopole and two loops [6]. These radars exploit the directional properties of the conventional MCL antenna to determine bearing using the multiple signal classification (MUSIC) [7] algorithm. Because of the small size, this type of HFR has been widely used across the world.

Many studies of HFR surface current measurements have validated the capacity of the direction-finding HFR to remote sensing the ocean surface currents through comparisons with in situ current measurements, such as [8-12]. These studies demonstrated that there is a $7-20 \mathrm{~cm} / \mathrm{s}$ differences between the current measurements derived from the directionfinding HFRs and those from the in situ instrument. And the DOA determination error is the dominant contributor to these differences.

To alleviate the DOA determination error and improve the performance of direction-finding HFR system in current measuring, a dual MCL antenna array, composed of two MCL elements, was investigated in this study. Because of the special structure of this antenna system, only the MUSIC direction-finding algorithm can be used to determine the DOA of the sea echoes. Thus, the relationship between the 
DOA estimation performance and the relative position of the two MCL elements was investigated, and we found that the spacing between the two antenna elements is not limited to the conventional half-wavelength condition due to the amplitude directional properties of the MCL element. And the simulation results suggested that the dual MCL antenna array has a better performance than the conventional single MCL antenna in current measuring, especially for the complex current pattern. Moreover, this performance improvement of the dual MCL antenna array relative to the conventional MCL antenna is also confirmed by the field experiment.

\section{Dual Monopole-Cross-Loop Antenna Array}

A MCL antenna array is composed of multiple MCL antenna elements. And a MCL antenna element consists of a monopole and two orthogonal loops. The antenna pattern of the MCL antenna element can be expressed as

$$
\begin{aligned}
& a_{1}(\theta)=1, \\
& a_{2}(\theta)=\cos \theta, \\
& a_{3}(\theta)=\sin \theta,
\end{aligned}
$$

where $a_{1}, a_{2}$, and $a_{3}$ represent the monopole and the two loops. Thus, the steering vector for signal coming from the direction of $\theta$ is $\mathbf{A}(\theta)=\left[a_{1}(\theta), a_{2}(\theta), a_{3}(\theta)\right]^{T}$. On the other hand, the steering vector for uniform liner array consisting of identical omnidirectional element with a spacing of $d$ can be given as

$$
\mathbf{A}_{\mathrm{u}}(\theta)=\left[1, e^{j \beta(\theta)}, e^{j 2 \beta(\theta)}, \ldots, e^{j(m-1) \beta(\theta)}\right]^{T},
$$

where $m$ is the number of antenna elements; $\beta(\theta)=-2 \pi d$ $\sin \theta / \lambda$ is the phase shift for adjacent elements; and $\lambda$ is the wavelength. Actually, the MCL antenna array is a synthesis of the MCL antenna and the linear antenna array. Thus, the steering vector of this hybrid array can be written as

$$
\mathbf{A}_{v}(\theta)=\left[\mathbf{A}^{T}(\theta), e^{j \beta(\theta)} \mathbf{A}^{T}(\theta), e^{j 2 \beta(\theta)} \mathbf{A}^{T}(\theta), \ldots, e^{j(m-1) \beta(\theta)} \mathbf{A}^{T}(\theta)\right]^{T} .
$$

For a dual MCL array, the steering vector can be reduced to

$$
\begin{aligned}
\mathbf{A}_{\mathrm{D}}(\theta) & =\left[\mathbf{A}^{T}(\theta), e^{j \beta(\theta)} \mathbf{A}^{T}(\theta)\right]^{T} \\
& =\left[1, \cos \theta, \sin \theta, e^{j \beta(\theta)}, e^{j \beta(\theta)} \cos \theta, e^{j \beta(\theta)} \sin \theta\right]^{T} .
\end{aligned}
$$

The amplitude directional properties denoted by $\mathbf{A}_{\mathrm{u}}^{T}(\theta)$ in (3) and (4) result in the difference between MCL array and the conventional uniform linear array. In conventional uniform linear array, the spacing, $d$, must be no more than half wavelength. If the spacing goes against this criterion, there will be ambiguity in DOA determination, because the condition leading to ambiguity is $\mathbf{A}_{\mathrm{u}}(\theta)=\mathbf{A}_{\mathrm{u}}\left(\theta^{\prime}\right)$ with $\theta \in\left[-90^{\circ}, 90^{\circ}\right], \theta^{\prime} \in\left[-90^{\circ}, 90^{\circ}\right]$, and $\theta \neq \theta^{\prime}$, that is, $e^{j n \beta(\theta)}=$ $e^{j n \beta\left(\theta^{\prime}\right)}$, with $n$ being an integer. Thus, the ambiguity condition is equivalent to

$$
\beta(\theta)-\beta\left(\theta^{\prime}\right)=\frac{2 \pi d \sin \theta}{\lambda}-\frac{2 \pi d \sin \theta^{\prime}}{\lambda}=2 k \pi,
$$

where $k$ is an integer. And it is straightforward to rewrite (5) as

$$
\frac{d}{\lambda}=\frac{k}{\sin \theta-\sin \theta^{\prime}}
$$

If the absolute value of the right side in (6) is larger than $0.5, d / \lambda$ being smaller than 0.5 will result in no solutions for $\theta$ and $\theta^{\prime}$ in (6), that is, no ambiguity in DOA determination. On the contrary, if $d / \lambda$ is more than 0.5 , the ambiguity will appear. Therefore, the spacing of adjacent element in conventional uniform linear array has to be no more than half wavelength for avoiding ambiguity in DOA estimation. But this is not the case for the MCL array. The ambiguity condition for MCL array is $\mathbf{A}_{v}(\theta)=\mathbf{A}_{v}\left(\theta^{\prime}\right)$, which is equivalent to simultaneously satisfy the following:

$$
\begin{gathered}
e^{j n \beta(\theta)}=e^{j n \beta\left(\theta^{\prime}\right),} \\
\mathbf{A}(\theta)=\mathbf{A}\left(\theta^{\prime}\right), \\
\theta \in\left[0^{\circ}, 360^{\circ}\right], \\
\theta^{\prime} \in\left[0^{\circ}, 360^{\circ}\right], \\
\theta \neq \theta^{\prime} .
\end{gathered}
$$

Clearly, there is no $\theta$ and $\theta^{\prime}$ satisfying (7) due to the presence of $\mathbf{A}(\theta)=\mathbf{A}\left(\theta^{\prime}\right)$, so there is no ambiguity in DOA estimation even for arbitrary spacing of the adjacent elements and for 360-degree look angle space.

But the performance of direction-finding algorithm is always related to the configuration of the antenna array, so that investigation of the effects of the number of elements and the spacing of the MCL array on DOA estimation in terms of MUSIC direction-finding algorithm is significant. In fact, Stoica and Nehorai [13] have proven that the estimated DOA, $\widehat{\theta}$, in MUSIC for arbitrary antenna system is a Gaussian distribution with a mean value being equal to the actual DOA, $\theta$, and the variance given by

$$
\operatorname{var}(\widehat{\theta})=\frac{1}{2 N \cdot \mathrm{SNR} \cdot h(\theta)}\left[1+\frac{\mathbf{b}^{H}(\theta) \mathbf{b}(\theta)}{\mathrm{SNR}}\right]
$$

where $\theta$ is the actual DOA of the incident signal; $\mathbf{b}(\theta)$ is the steering vector; $N$ is the number of samples; SNR is signal-tonoise ratio; and $h(\theta)=\mathbf{d}^{H}(\theta)\left\{I-\mathbf{b}(\theta)\left[\mathbf{b}^{H}(\theta) \mathbf{b}(\theta)\right]^{-1} \mathbf{b}^{H}(\theta)\right\}$ $\mathbf{d}(\theta)$ with $\mathbf{d}(\theta)=d \mathbf{b}(\theta) / d \theta$. Thus, in the case of a MCL array composed of $m$ identical MCL elements with a uniform spacing of $d$, we have $\mathbf{b}(\theta)=\mathbf{A}_{v}(\theta)$ and $\mathbf{d}(\theta)=d \mathbf{A}_{v}(\theta) / d(\theta)$, which gives 


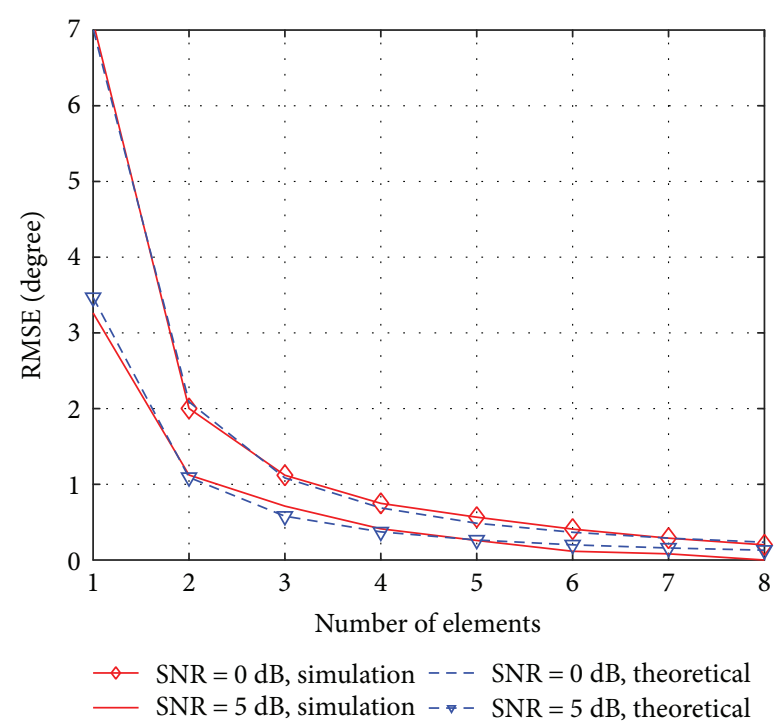

Figure 1: The root-mean-square error (RMSE) of the DOA estimation versus the number of antenna element with the signal coming from $30^{\circ}$ and the spacing between adjacent elements being equal to half wavelength.

$\operatorname{var}^{v}[\hat{\theta}]=\frac{1}{2 N \cdot \mathrm{SNR}} \times\left(1+\frac{1}{2 m \cdot \mathrm{SNR}}\right) \times\left[\frac{6}{6 m+m\left(m^{2}-1\right)\left[\beta^{\prime}(\theta)\right]^{2}}\right]$,

where $\beta^{\prime}(\theta)=d \beta(\theta) / d \theta=2 \pi d \cos \theta / \lambda$. Figure 1 shows the DOA estimation error varying with the number of the MCL elements. The simulation results shown in this figure are achieved by a Monte-Carlo simulation of 300 independent runs with 50 snapshots for each trial, while the theoretical results are directly calculated by (9) with the same parameters used in the simulation. To make the unit of the results obtained from (9) being the same as the unit of the DOA, the square root of the the variance (standard deviation), which is equal to the root-mean-square error due to the mean value of the DOA estimation error being zero, is used in Figure 1. These results displayed in Figure 1 indicate that the DOA estimation error decreases with the antenna element increases expectedly. Besides, the rate of the decrease gradually slows down and, eventually, the DOA estimation accuracy levels off at almost the same level for different signal-to-noise ratio (SNR). Taking the occupation of space of an antenna system into consideration, we suggest that an MCL antenna array composed of two or three MCL elements is an optimal scheme in practice. But in this study, we only focus on dual MCL antenna array composed of only two MCL elements.

In the case of the dual MCL antenna array, (9) can be reduced to

$\operatorname{var}^{\mathrm{D}}[\widehat{\theta}]=\frac{1}{2 N \cdot \mathrm{SNR}} \times\left(1+\frac{1}{4 \cdot \mathrm{SNR}}\right) \times\left[\frac{1}{2+(2 \pi d \cos \theta / \lambda)^{2}}\right]$.

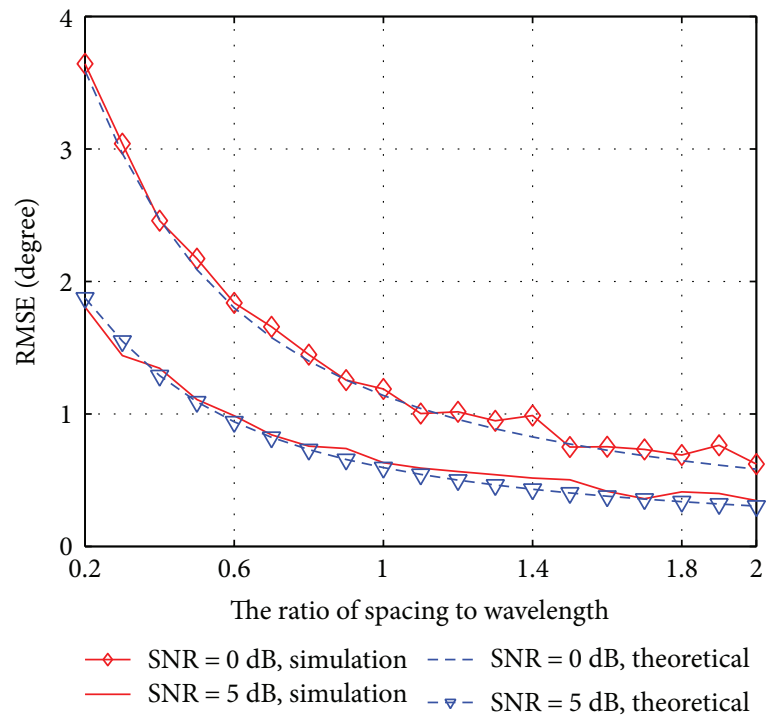

Figure 2: The RMSE value of the DOA estimation versus the spacing in dual MCL antenna array with signal coming from $30^{\circ}$.

In the foregoing analysis, we have concluded that the spacing for the MCL array can be arbitrary value. Thus, discussing the effect of the spacing on the DOA estimation performance for the dual MCL array is necessary. Figure 2 shows the RMSE value of the DOA estimation varying with the spacing. The simulation results are obtained from Monte-Carlo simulation with the same independent runs and snapshots as in Figure 1. The theoretical results are computed as (10). From this figure, we can clearly see that the RMSE values decrease with the spacing increase. But the decrease is very limited especially for a high SNR. These results guide us that the spacing can be relaxed, and the deployment of the dual MCL antenna array is relatively convenient for real applications. In this study, the parameter of this spacing was set to be equal to the wavelength. With this setting, Figure 3 shows the comparison of the DOA estimation performance between the dual MCL antenna array and the conventional single MCL antenna system. The performance of the conventional single MCL antenna system can be expressed as

$$
\operatorname{var}^{\mathrm{C}}[\widehat{\theta}]=\frac{1}{2 \mathrm{~N} \cdot \mathrm{SNR}} \times\left(1+\frac{1}{2 \cdot \mathrm{SNR}}\right)
$$

Comparing (11) with (10), we can clearly know that $\operatorname{var}^{\mathrm{C}}[\hat{\theta}]$ is always more than $\operatorname{var}^{\mathrm{D}}[\hat{\theta}]$, which indicates that the performance of the dual MCL array is superior to the single MCL antenna. And Figure 3, showing the DOA estimation error for dual and single MCL antenna system, also clearly indicates this result. Thus, using two MCL antenna elements to jointly observe sea surface current is an advisable scheme.

\section{Ocean Surface Current Extraction}

3.1. Extraction Current Mappings with MUSIC Algorithm. To obtain the current velocity mappings, radar signal processing 


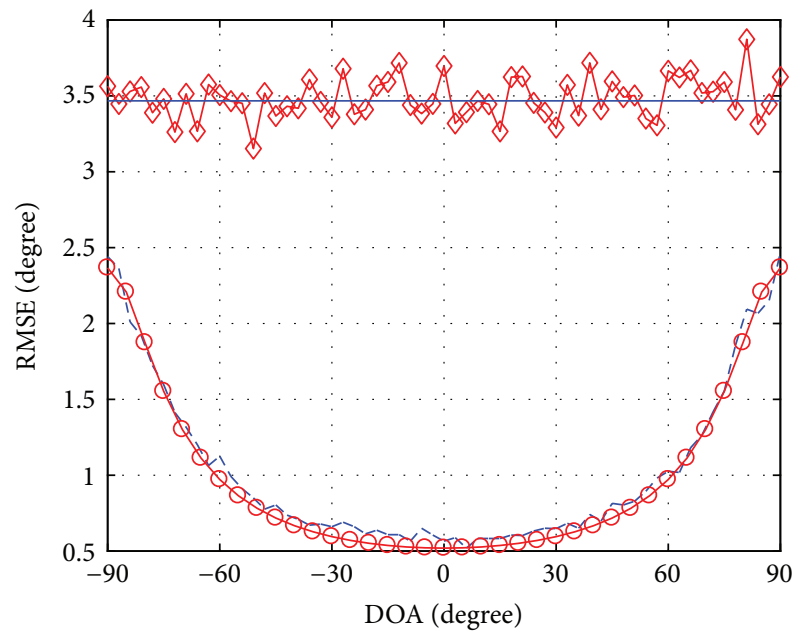

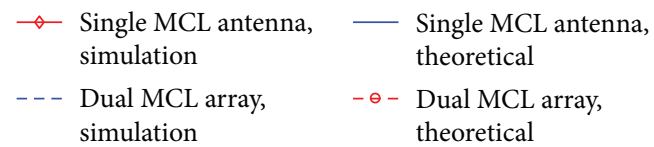

Figure 3: DOA estimation errors for the conventional single MCL antenna and the dual MCL antenna array vary with the DOA with a signal to noise ratio of $5 \mathrm{~dB}$.

involves the resolving of the range, Doppler, and direction of arrival (DOA) of the backscattered signals. The time delay of this backscattered signal is used to measure the distance of the patch to the radar, while the frequency difference between observed Doppler shift and expected position of the Bragg peaks is used to deduce the current velocity. The DOA is determined either by the MUSIC or the beam forming. But only the MUSIC is able to address the DOA of the sea echo received by a conventional or dual MCL antenna system due to the special geometry of the antenna system.

The DOA estimation using MUSIC was introduced by Schmidt [7]. This method determines a noise space via diagonalizing the covariance matrix formed from the multichannel signals, and it determines the signal bearing by finding the smallest projection of the steering vectors on that noise space. For the conventional MCL antenna, the covariance matrix is formed from the signals received by the monopole and the two loops, while the covariance matrix is derived from the two MCL antenna array for the dual MCL antenna system. After diagonalizing the covariance matrix, we have to determine the number of sources involved in current samples. Barrick and Lipa $[14,15]$ have proposed a hypothesis-testing method to determine the number of sources for the conventional MCL antenna system. In this study, we also use this hypothesis-testing method to determine the number of sources when we extract current mappings in conventional MCL antenna system with the three parameters: eigenvalue ratio, signal power ratio, and diagonal ratio, being 40, 20, and 3, respectively. Extraction current mappings involved in dual MCL array also use the way of hypothesis testing to determine the number of sources. We first test the number of sources of three with the same approach

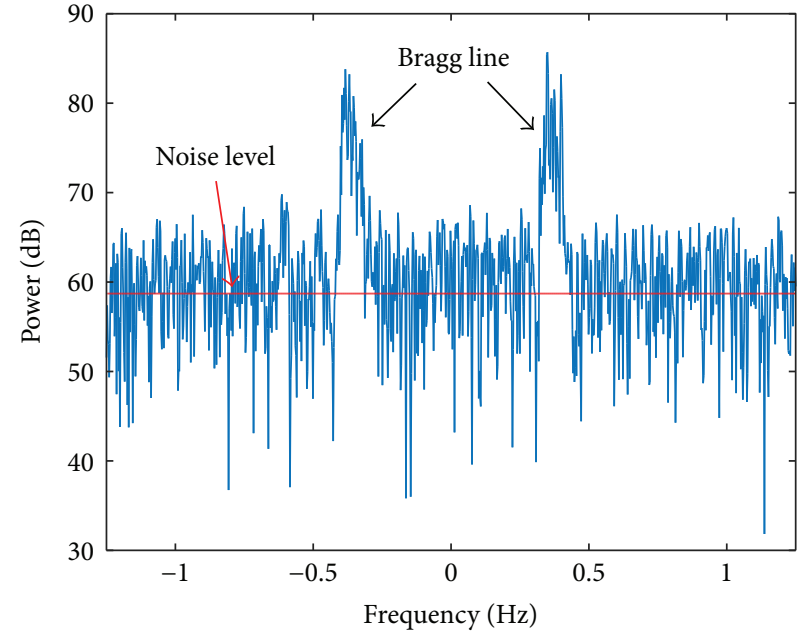

Figure 4: An example of the Doppler spectrum for monopole channel in the simulations.

used in the conventional MCL antenna system with the three parameters: eigenvalue ratio, signal power ratio, and diagonal ratio, being 50,20, and 2, respectively. If this testing indicates that the number of sources is not three, we then do testing the number of sources of two with the same approach and parameters involved in the conventional MCL antenna system. If this testing of two sources also indicates that the number of sources is not two, we regard these samples as single source in DOA estimation process.

3.2. Simulation on Current Retrieval. To validate the performance improvement of this antenna array to the conventional MCL antenna, we carried out simulations on retrieving current velocities and compared the performance of these two antenna systems. These simulations generated the complex time series for each antenna channel received from a range cell (which is a circular annulus defined by the range from the radar) based on the method adopted by Wang and Gill [16]. Practically, these time series are induced by backscatter from the sea due to the first-order Bragg scattering. In this study, we investigated three radial current scenarios in this range cell: a uniform current flowing parallel to shore (which is a representation of single DOA for any current velocity), a parabola-shaped current (which involves dual DOAs for some current velocity), and a current jet superimposed with a uniform parallel-to-shore current (which implies three DOAs for some current velocity). In addition, we assumed a uniform parallel-to-shore wind and its speed, duration, and fetch were assumed to be sufficient for fully developed Bragg scattering waves having a broad cardioid directional distribution of spectral energy [17]. The additive Gaussian noise was added to these time series with an SNR of $20 \mathrm{~dB}$ (defined as the ratios of the Bragg lines power to the noise level, say Figure 4). Then, a Doppler spectrum is obtained by performing FFT on each time series. An example of the Doppler spectrum in our simulation is shown in Figure 4. 


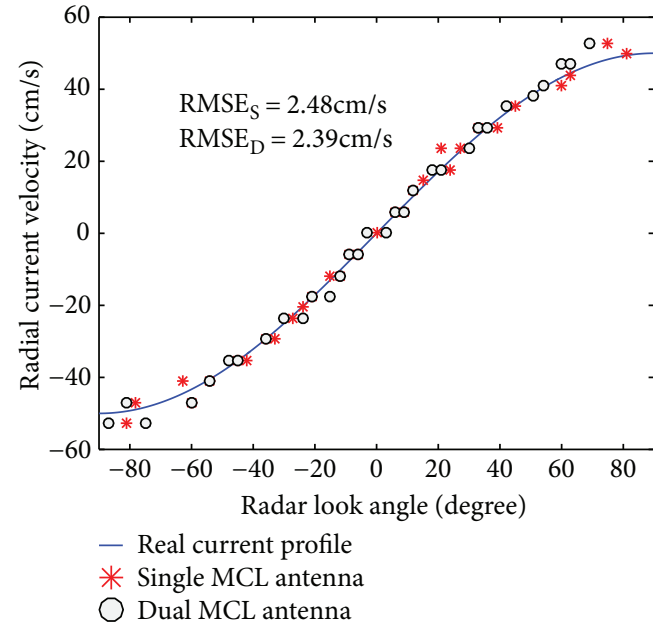

(a)

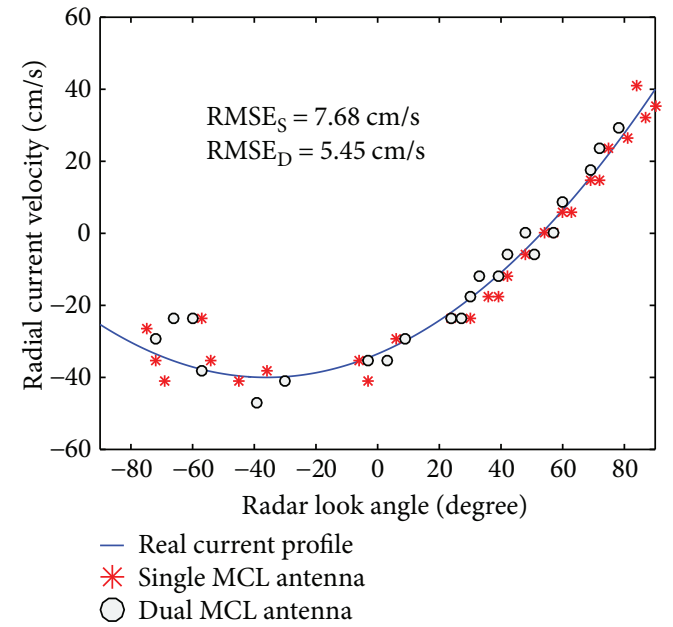

(b)

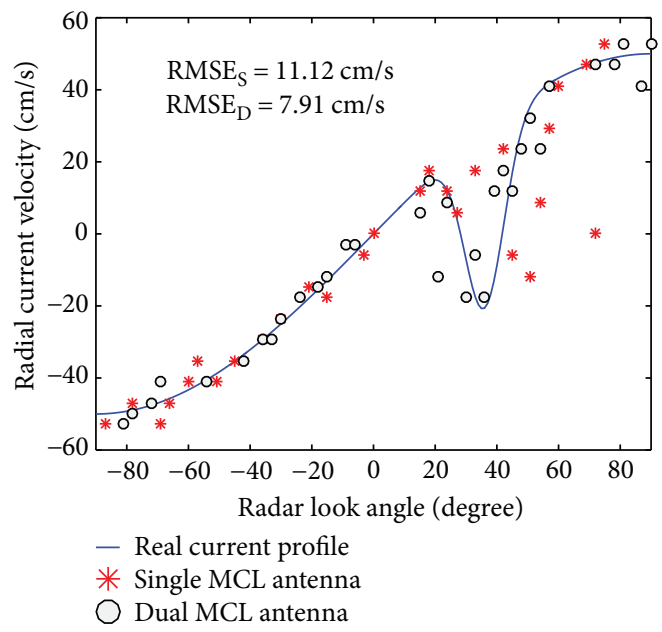

(c)

Figure 5: Examples of the radial current profiles (solid line) with measurements from conventional single MCL antenna (star) and those from dual MCL antenna array (circle). The RMSE $\mathrm{S}_{\mathrm{S}}$ and $\mathrm{RMSE}_{\mathrm{D}}$ are the RMSE values of current comparison results for conventional single MCL antenna and the dual MCL antenna array, respectively.

For each radial current profile scenarios, we performed 100 independent runs for both single MCL antenna and dual MCL antenna systems. The indicator of the root-meansquare error (RMSE) was adopted to assess the accuracy of the retrieved radial current velocities. Examples for the mentioned three current profiles and the estimated radial currents, as well as the RMSE values for all the 100 runs, are shown in Figure 5. From this figure, we can conclude that the dual MCL antenna configuration has a better performance than the conventional single MCL antenna system in current mapping, especially for the complex current profile. Surely, the current pattern displayed in Figure 5(c) is unfair for the conventional MCL antenna system because the current velocities ranging $-20 \mathrm{~cm} / \mathrm{s}$ to $10 \mathrm{~cm} / \mathrm{s}$ have three DOAs, which is beyond the angular solving capacity of single MCL element. Fortunately, this scenario is rare encounter in practice, so that the conventional single MCL antenna system can often retrieve acceptable current mappings. But this scenario, sometimes, may indeed be present in reality.

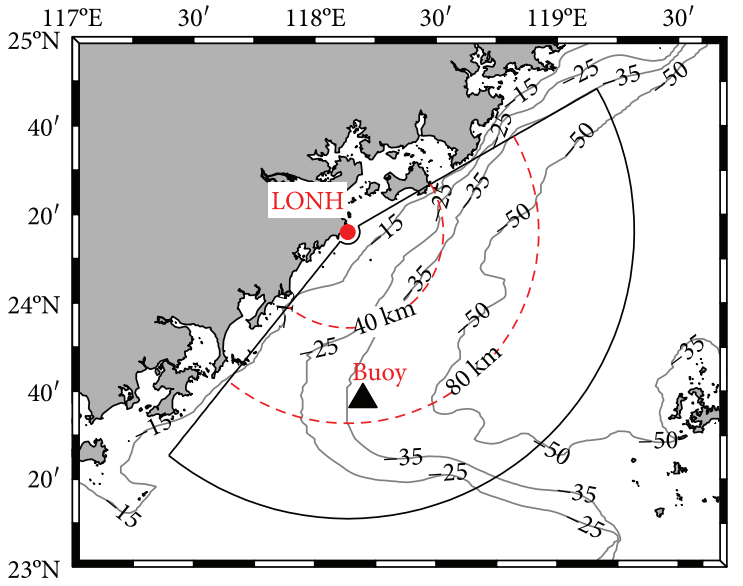

FIgURe 6: Map of the deployment of the radar (red dot) and the buoy (black triangle). The buoy is at a distance about $75 \mathrm{~km}$ from the radar site. The gray thin lines are the isobaths. The fanwise area denotes the radar detection region. 


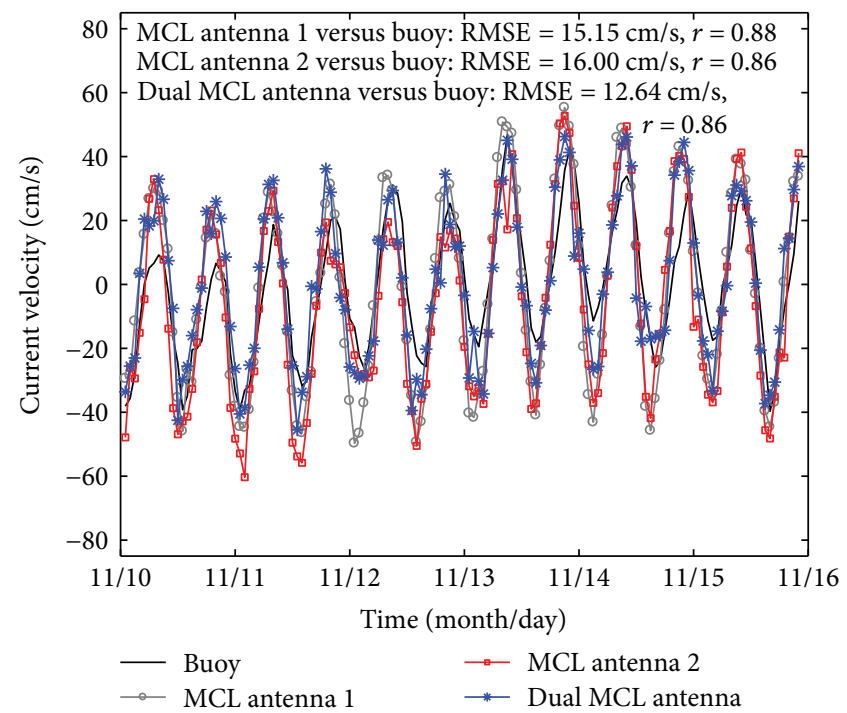

(a)

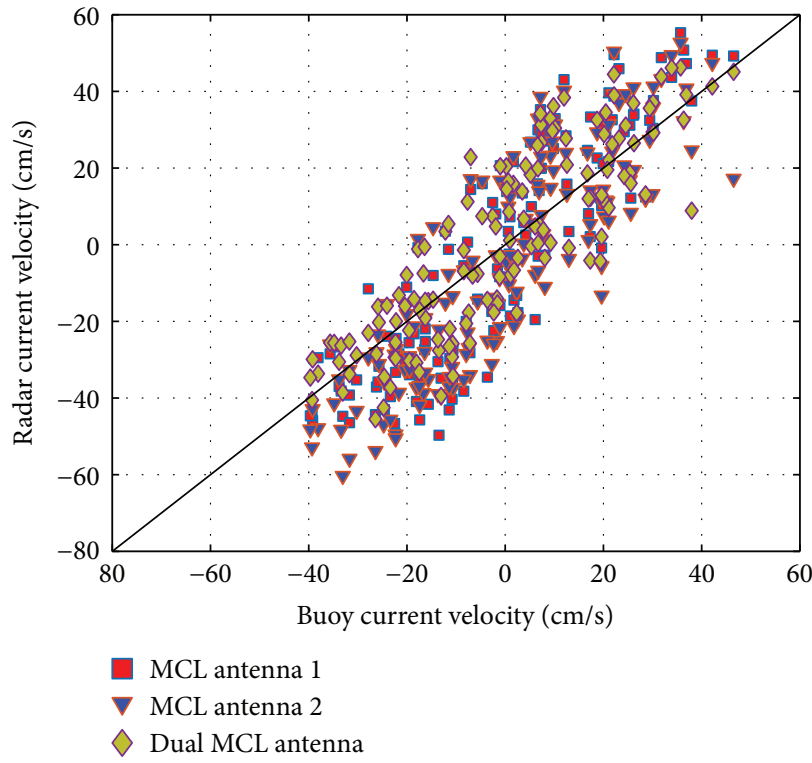

(b)

FIgURE 7: Radial current velocity comparisons. (a) Time series of the buoy-derived radial current velocity and that derived by LONH radar site with the MCL antenna 1, MCL antenna 2, and the dual MCL antenna system. Statistics, correlation coefficient $(r)$, and root-mean-square error (RMSE) of each radar-ADCP current comparison pairs are shown on the top of (a). (b) Scatter plot of the radial current velocities.

\section{Field Data Evaluation}

To assess the performance improvement of the dual MCL antenna system for practical radial current velocity retrieval, a field experiment was conducted in November 2015. In the experiment, an Ocean State Measuring and Analyzing Radar type S (OSMAR-S) radar system was deployed on the coast of Fujian province, chain, as illustrated in Figure 6. The radar emitted a frequency-modulated interrupting continuous waveform (FMICW) signal toward the ocean surface with a center frequency of $13 \mathrm{MHz}$. The bandwidth of the radar was $60 \mathrm{kHz}$ which theoretically produced a $2.5 \mathrm{~km}$ range resolution. Two MCL antennas with a spacing equaling to the wavelength of the radar-transmitted signal were set for receiving the echo scattered from the ocean surface. Thus, we could simultaneously sample the sea echoes in six channels. And both the first three channels (antenna 1) and the rest three channels (antenna 2) are a conventional MCL antenna, which is able to extract the ocean surface maps. The samples in these channels were collected at an interval of $0.54 \mathrm{~s}$. And a 512-point FFT was performed on these samples to yield a coherent integration time of about $276.5 \mathrm{~s}$ or a current velocity resolution of $4.1 \mathrm{~cm} / \mathrm{s}$.

The in situ measurements used in this study were from a buoy-mounted acoustic Doppler current profile (ADCP). The location of the buoy is about $75 \mathrm{~km}$ away from the radar site (Figure 6). This single-point ADCP provided current velocity every 10 minutes with a velocity resolution of $0.1 \mathrm{~cm} / \mathrm{s}$. The depth of the ADCP measurements used in this study is 6 meters under the water.

The radar-derived radial currents in this experiment for the two antenna configurations (one MCL antenna and dual MCL antenna array combining the two MCL antenna elements) were compared with the buoy measurements. In the current extraction process, the ideal antenna patterns were used and the two MCL antennas have been calibrated independently on the basis of the geometrical relationship for the three-channel collocated MCL antenna [3]. For the dual MCL antenna array, the channel errors between the two MCL antenna elements were also calibrated using known sources. The radial currents derived by single MCL antenna and dual MCL antenna are shown in Figure 7 where comparison statistics are also provided. Comparison of the radial currents derived from antenna 1 and antenna 2 with the ADCP measurements yields RMSE values of $15.15 \mathrm{~cm} / \mathrm{s}$ and $16.00 \mathrm{~cm} / \mathrm{s}$, respectively. While the RMSE value for the radial current velocities derived from the dual MCL antenna configuration reduces to $12.64 \mathrm{~cm} / \mathrm{s}$. This really suggests the improvement of the dual MCL antenna system to the single MCL antenna. However, the correlation coefficient between the dual MCL antenna deduced radial currents and those observed by ADCP is nearly the same with that of either of the single MCL antennas.

Another indicator of the quality of the radial currents extracted from HF radar is the temporal coverage rate. The spatial distributions of the temporal coverage rates for radial currents derived from antenna 1 , antenna 2, and dual MCL antenna are achieved and shown in Figure 8. These temporal coverage rate maps are calculated for each radial sector as the total number of the valid radial current estimators divided by the total number of the radial current maps. From Figure 8, we can see an obvious consistency that the temporal coverage rate has a decrease with distance from the radar site origin. But the radial current derived from dual antenna with a temporal coverage rate greater than 0.6 extends to a much larger area than either that from single conventional MCL 


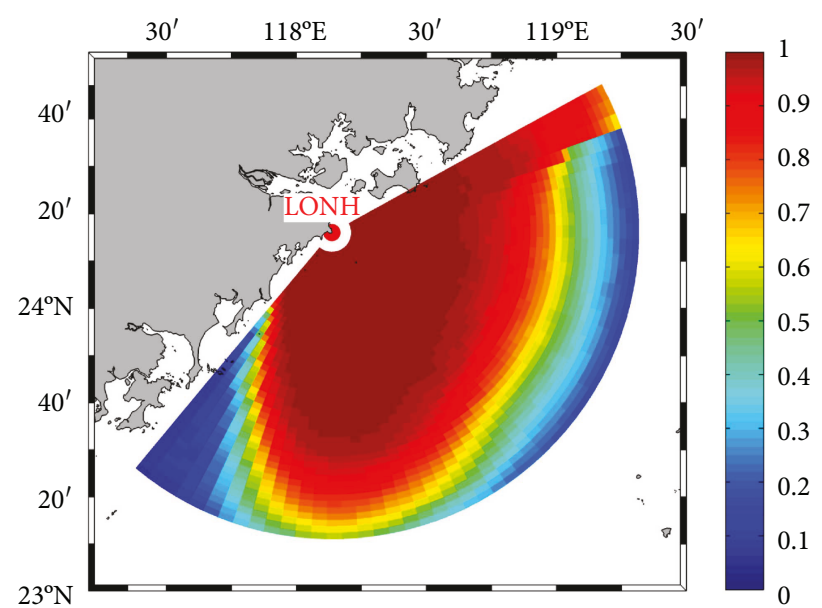

(a)

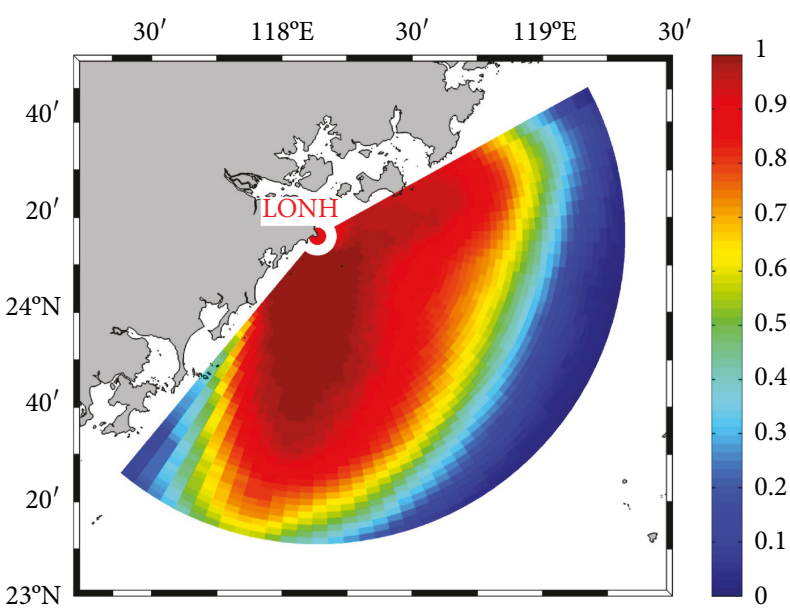

(b)

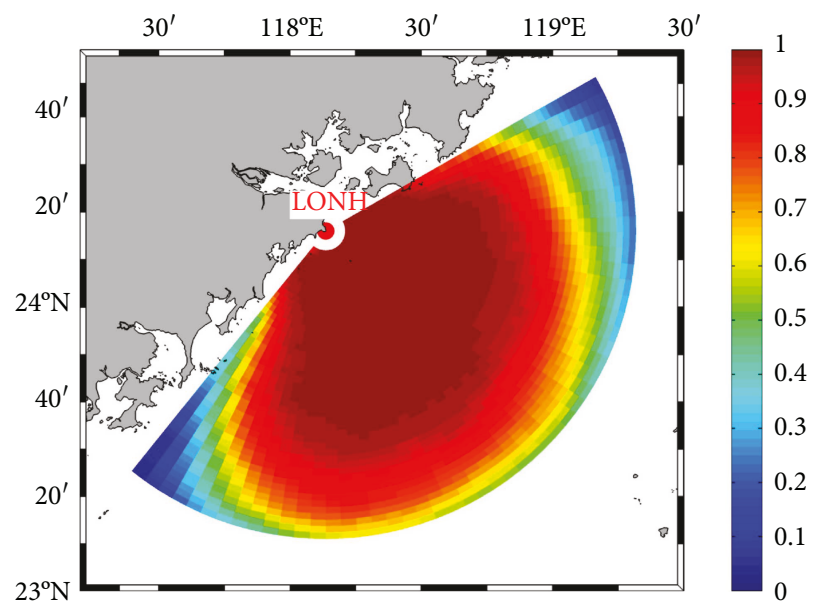

(c)

FIGURE 8: Temporal coverage rate of valid radial currents for LONH site with MCL antenna 1 (a), MCL antenna 2 (b), and dual MCL antenna system (c).

antennas. Thus, the quality of the ocean surface currents observed by a dual MCL antenna is really better than the conventional single MCL antenna system. Due to the fact that temporal coverage rate of the retrieved current maps is interested by the current-dataset user, using the dual MCL array to observe oceanic surface current field is an advisable approach.

\section{Conclusions}

In this study, we have investigated the performance improvement of the dual MCL antenna array to the conventional single MCL antenna. We analyzed the effects of the MCL antenna array parameters on DOA estimation. An analysis of pointing error related to the number of MCL elements shows that a MCL antenna array consisted of two or three MCL elements is an optimal scheme in practice. And the examination of the spacing between the MCL elements suggests that the spacing between the two antenna elements is permitted to exceed the conventional half wavelength limitation for the linear phased array. Simulation on current extraction for a dual MCL antenna array with wavelength spacing indicates that this antenna system can improve the radar performance in current retrieval relative to the conventional single MCL antenna system, especially for the complex current profile. Moreover, this performance improvement has been validated by the field experiment. The results of the field experiment show that the RMSE value of the radial current retrieved by conventional single MCL antenna was larger than $15 \mathrm{~cm} / \mathrm{s}$ with respect to the ADCP measurements, but it decreased to $12.64 \mathrm{~cm} / \mathrm{s}$ when the dual MCL antenna array was adopted in current observation.

\section{Conflicts of Interest}

The authors declare that there is no conflict of interest regarding the publication of this paper.

\section{Acknowledgments}

This work was supported by the National Natural Science Foundation of China (NSFC) under Grant 61371198 and the National Special Program for Key Scientific Instrument and Equipment Development of China under Grant 
2013YQ160793. The authors would like to thank the personnel of Wuhan Device Electronic Technology Co. Ltd. for carrying out the experiment.

\section{References}

[1] J. D. Paduan and L. Washburn, "High-frequency radar observations of ocean surface currents," Annual Review of Marine Science, vol. 5, no. 1, pp. 115-136, 2013.

[2] K. W. Gurgel, G. Antonischki, H. H. Essen, and T. Schlick, "Wellen radar (WERA): a new ground-wave HF radar for ocean remote sensing," Coastal Engineering, vol. 37, no. 3-4, pp. 219-234, 1999.

[3] B. Lipa and D. Barrick, "Least-squares methods for the extraction of surface currents from codar crossed-loop data: application at ARSLOE," IEEE Journal of Oceanic Engineering, vol. 8, no. 4, pp. 226-253, 1983.

[4] H. Zhou and B. Wen, Portable High Frequency Surface Wave Radar OSMAR-S, Springer International Publishing, Cham, 2015.

[5] H. Zhou and B. Wen, "Wave height extraction from the firstorder bragg peaks in high-frequency radars," IEEE Geoscience and Remote Sensing Letters, vol. 12, no. 11, pp. 2296-2300, 2015.

[6] A. R. Carr, "Three-element antenna formed of orthogonal loops mounted on a monopole," US Patent 4,433,336, 1984.

[7] R. Schmidt, "Multiple emitter location and signal parameter estimation," IEEE Transactions on Antennas and Propagation, vol. 34, no. 3, pp. 276-280, 1986.

[8] S. Cosoli, A. Mazzoldi, and M. Gačić, "Validation of surface current measurements in the northern Adriatic sea from high-frequency radars," Journal of Atmospheric and Oceanic Technology, vol. 27, no. 5, pp. 908-919, 2010.

[9] Y. Liu, R. H. Weisberg, and C. R. Merz, "Assessment of CODAR seasonde and WERA HF radars in mapping surface currents on the west Florida shelf," Journal of Atmospheric and Oceanic Technology, vol. 31, no. 6, pp. 1363-1382, 2014.

[10] P. Lorente, S. Piedracoba, and E. A. Fanjul, "Validation of high-frequency radar ocean surface current observations in the NW of the Iberian peninsula," Continental Shelf Research, vol. 92, pp. 1-15, 2015.

[11] A. Kalampokis, M. Uttieri, P. M. Poulain, and E. Zambianchi, "Validation of HF radar-derived currents in the Gulf of Naples with lagrangian data," IEEE Geoscience and Remote Sensing Letters, vol. 13, no. 10, pp. 1452-1456, 2016.

[12] Y. Lai, H. Zhou, J. Yang, Y. Zeng, and B. Wen, "Submesoscale eddies in the Taiwan Strait observed by high-frequency radars: detection algorithms and eddy properties," Journal of Atmospheric and Oceanic Technology, vol. 34, no. 4, pp. 939-953, 2017.

[13] P. Stoica and A. Nehorai, "MUSIC, maximum likelihood, and cramer-rao bound," IEEE Transactions on Acoustics, Speech, and Signal Processing, vol. 37, no. 5, pp. 720-741, 1989.

[14] D. E. Barrick and B. J. Lipa, "Evolution of bearing determination in HF current mapping radars," Oceanography, vol. 10, pp. 72-75, 1997.

[15] D. E. Barrick and B. J. Lipa, "Radar angle determination with MUSIC direction finding,” US Patent 5,990,834, 1999.
[16] W. Wang and E. W. Gill, "Evaluation of beamforming and direction finding for a phased array HF ocean current radar," Journal of Atmospheric and Oceanic Technology, vol. 33, no. 12, pp. 2599-2613, 2016.

[17] M. S. Longuet-Higgins, "Observations of the directional spectrum of sea waves using the motions of a floating buoy," in Ocean Wave Spectra: Proceedings of a Conference, PrenticeHall, pp. 111-136, 1961. 


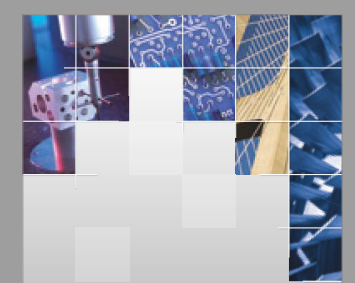

\section{Enfincering}
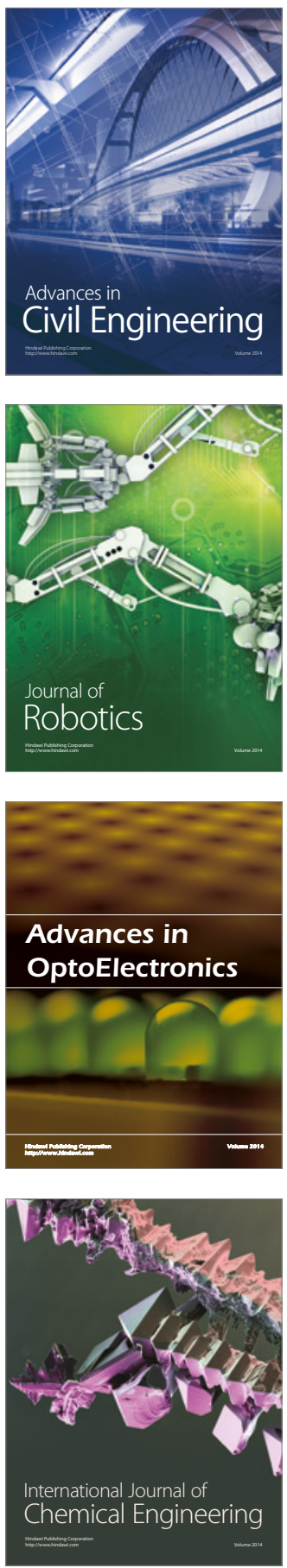

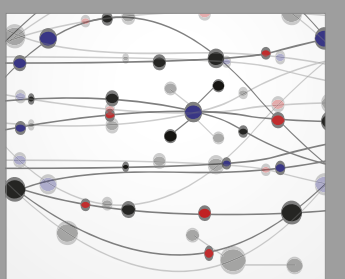

The Scientific World Journal

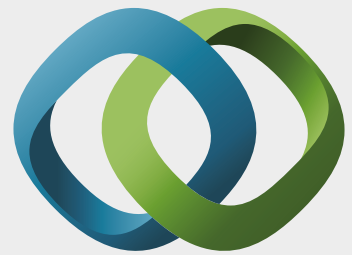

\section{Hindawi}

Submit your manuscripts at

https://www.hindawi.com
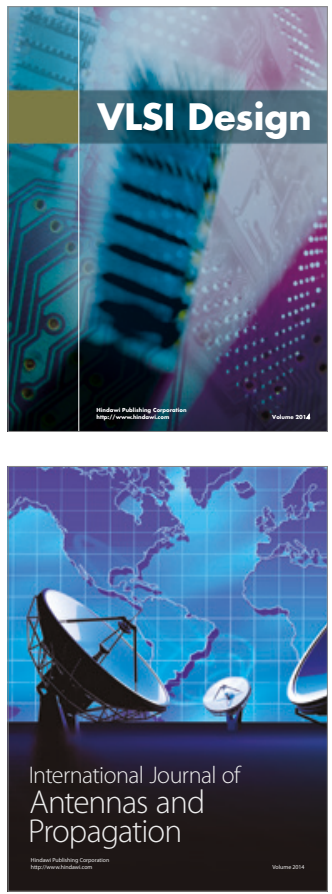

\section{Rotating}

Machinery
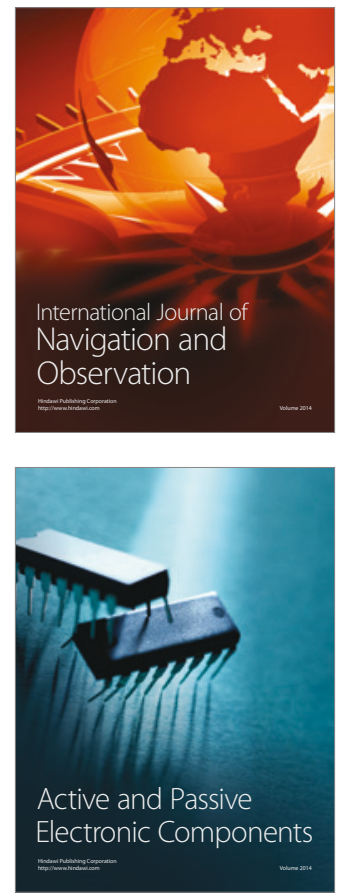
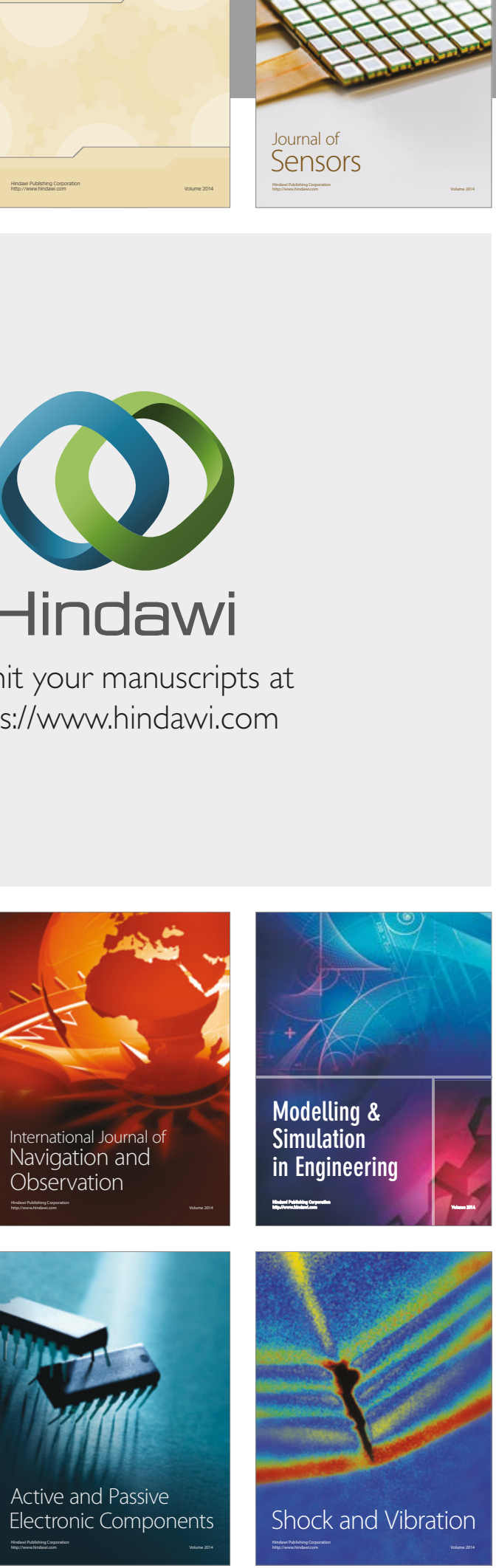
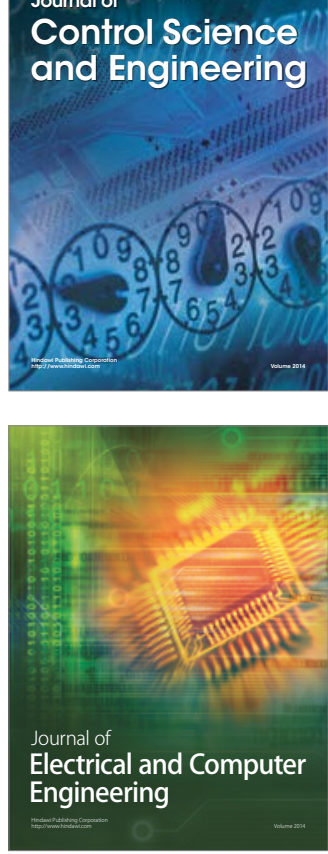

Distributed

Journal of

Control Science

and Engineering
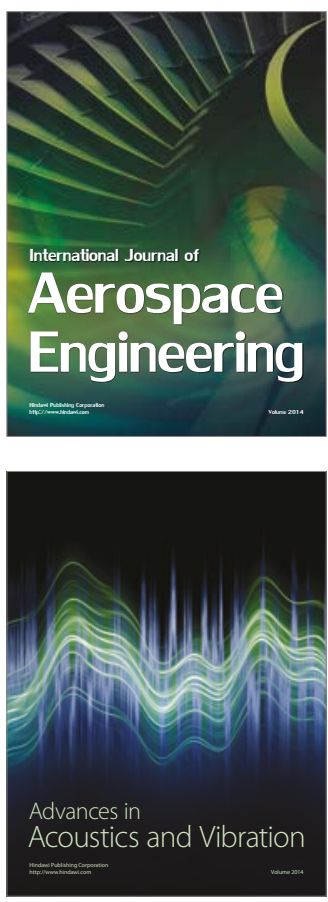

Sensor Networks 